\title{
Parton-shower effects in Higgs production via vector-boson fusion
}

\author{
Barbara Jäger ${ }^{1}$, Alexander Karlberg ${ }^{2}$, Simon Plätzer ${ }^{3,4}$, Johannes Scheller ${ }^{1}$, Marco Zaro $^{5, \mathrm{a}}$ (i) \\ ${ }^{1}$ Institute for Theoretical Physics, University of Tübingen, Auf der Morgenstelle 14, 72076 Tübingen, Germany \\ 2 Rudolf Peierls Centre for Theoretical Physics, University of Oxford, Clarendon Laboratory, Parks Road, Oxford OX1 3PU, UK \\ ${ }^{3}$ Particle Physics, Faculty of Physics, University of Vienna, 1090 Wien, Austria \\ ${ }^{4}$ Erwin Schrödinger Institute for Mathematics and Physics, University of Vienna, 1090 Wien, Austria \\ ${ }^{5}$ INFN Sezione di Milano \& TifLab, Via Celoria 16, 20133 Milan, Italy
}

\begin{abstract}
We present a systematic investigation of partonshower and matching uncertainties of perturbative origin for Higgs-boson production via vector-boson fusion. To this end we employ different generators at next-to-leading order QCD accuracy matched with shower Monte Carlo programs, PYTHIA8, and HERWIG7, and a next-to-next-to-leading order QCD calculation. We thoroughly analyse the intrinsic sources of uncertainty within each generator, and then compare predictions among the different tools using the respective recommended setups. Within typical vector-boson fusion cuts, the resulting uncertainties on observables that are accurate to next-to-leading order are at the $10 \%$ level for rates and even smaller for shapes. For observables sensitive to extra radiation effects uncertainties of about $20 \%$ are found. We furthermore show how a specific recoil scheme is needed when PYTHIA8 is employed, in order not to encounter unphysical enhancements for these observables. We conclude that for vector-boson fusion processes an assessment of the uncertainties associated with simulation at next-toleading order matched to parton showers based only on the variation of renormalisation, factorisation and shower scales systematically underestimates their true size.
\end{abstract}

\section{Introduction}

After the discovery of a Higgs boson compatible with the prediction of the Standard Model (SM) of elementary particles at the CERN Large Hadron Collider (LHC) by the ATLAS and CMS experiments [1,2], Higgs physics has entered the era of precision physics. While all measurements completed so far consolidate the SM hypothesis, only a comprehensive analysis of the new boson's properties will reveal whether deviations from the expectation leave room for new physics

\footnotetext{
a e-mail: marco.zaro@mi.infn.it (corresponding author)
}

in the experimentally accessible domain. The precise determination of the Higgs boson's couplings to other elementary particles, spin, and $\mathrm{CP}$ properties is thus of paramount importance.

A particularly clean environment for the necessary measurements at the LHC is provided by the vector-boson fusion (VBF) production mode where the Higgs boson is produced by two scattering partons in association with two hard jets (often referred to as tagging jets) in the forward and backward regions of the detector via the exchange of weak massive gauge bosons. Because of the colour-singlet nature of this mechanism, little extra jet activity occurs between the two tagging jets, in the central rapidity region of the detector. These features are of great relevance for separating the VBF signal from QCD background processes that typically exhibit entirely different jet distributions.

Precise measurements can unfold their potential only if matched by equally accurate theoretical predictions. Calculations of the highest accuracy are therefore mandatory in the analysis of VBF data obtained by the experimental LHC collaborations. We note that already now theoretical uncertainties are becoming a bottleneck in Higgs precision studies at the LHC. For instance, in the recent Higgs-combination study by the ATLAS collaboration [3], theory uncertainties are a dominant source of uncertainty in the VBF channel, exceeding statistical and experimental uncertainties. While the QCD corrections to Higgs production via VBF at next-toleading order (NLO) accuracy have been known for almost 30 years for inclusive cross sections [4] and for almost 20 years for differential distributions with realistic selection cuts [5,6] in the form of flexible parton-level Monte Carlo programs, NLO electroweak corrections have first been presented only later on in Ref. [7] and found to be of almost the same size as the NLO-QCD corrections. Several implementations of VBF-induced Higgs-boson production in programs allowing for a matching with parton-shower programs at NLO-QCD 

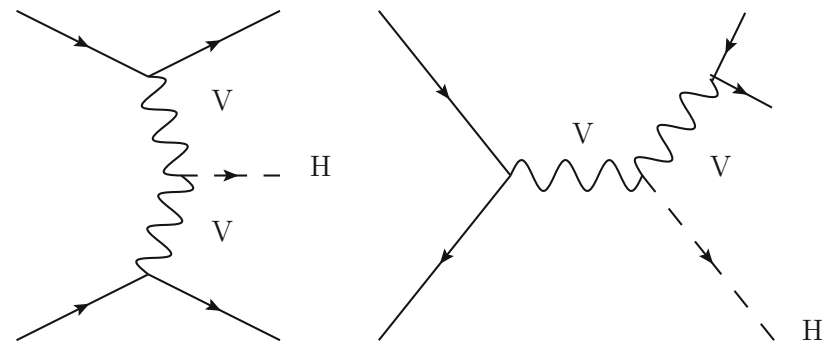

Fig. 1 Representative diagrams for electroweak production of a $H+$ 2 jet final state

accuracy (in the following referred to as NLO + PS accuracy) are available [8-10]. More recently, the fixed order next-tonext-to-leading order (NNLO) QCD corrections have been computed, again first for the fully inclusive case $[11,12]$ and later on differentially $[13,14]$. These corrections have been found to be small, but not negligible, for differential distributions in the presence of VBF specific cuts. Residual scale uncertainties are tiny at this order in QCD and can be further reduced by the consideration of the next-to-next-to-next-toleading order $\left(\mathrm{N}^{3} \mathrm{LO}\right) \mathrm{QCD}$ corrections [15]. Many of the quoted QCD calculations rely on the so-called "VBF approximation", which assumes the absence of colour exchange between the two fermion lines connected by the weak gauge bosons, and neglecting the interferences among $H+2$ jet final states produced via $s$-channel and $t$ - or $u$-channel topologies, c.f. Fig. 1. At NLO accuracy, the quality of this approximation has been explicitly tested in Ref. [7] and found to be very good once VBF-specific cuts are imposed that force the two tagging jets to be well separated from each other. The impact of different kind of corrections which violate this assumption has been investigated in Refs. [12,16] and recently in Ref. [17]. In all cases, it is found to be of the order of a percent at most.

Ideally, such accurate calculations are provided in the form of public Monte-Carlo programs that can be used by the experimental collaborations directly in their analyses. To make the most of these programs it is important to understand their systematic uncertainties and limitations, for instance due to underlying approximations. In order to provide a systematic assessment of the differences and similarities between commonly used public Monte-Carlo programs designed for VBF-induced Higgs boson production at NLO + PS accuracy, in this article we perform an in-depth comparison of key observables in VBF analyses using realistic input parameters and selection cuts for the respective implementations $[8-10,18]$ in the three generators MadGraph5_aMC@NLO[19,20], POWHEG-BOX[8], and HERWIG7[21, 22] VBFNLO+Herwig7/Matchbox[23,24] as well as HJet s+Herwig7/Matchbox[25].

We start with a description of the three generators considered in this study in Sect. 2, describe the setup of our analyses in Sect. 3, and discuss the main results of our study in Sectt. 4. We conclude with recommendations for the optimal use of the considered generators and a realistic assessment of the associated uncertainties in Sect. 5

\section{Generators}

\subsection{MadGraph5_aMC@NLO}

MadGraph5_aMC@NLO $[19,20]$ is a meta-code (i.e. a code that generates codes) which makes it possible to automatically simulate arbitrary scattering processes at NLO accuracy in the strong and electroweak couplings, either at fixed order or including matching to parton showers (when one considers only corrections of strong origin), using the MC@NLO method [26]. It employs the FKS subtraction method [27,28] (as automated in MADFKS [29,30]) for the local subtraction of IR singularities. One-loop amplitudes are evaluated by switching dynamically between two integral-reduction techniques, the OPP method [31] or a Laurent-series expansion [32], and tensor-integral reduction [33-35]. All such techniques have been automated in the module MADLOOP [36], which in turn links CUTTOOLs [37], NINJA [38,39], IREGI [40], or COLLIER [41], together with an in-house implementation of the OPENLOOPS technique [42]. Uncertainties associated with factorisation and renormalisation scales or partondistribution functions (PDFs) can be obtained without any approximation thanks to reweighting, at negligible additional CPU cost [43].

The simulation of Higgs production via VBF at NLOQCD accuracy can be performed with the following commands:

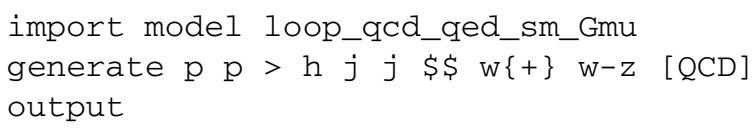

For the case of Higgs plus three jets production via VBF, one should simply add a $j$ to the generate command, i.e.:

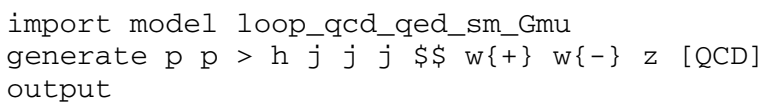

While results for the first process have been already published in Ref. [10] (although with rather old parton-shower programs), for the second they have been only briefly commented upon in Ref. [19]. In both cases, the \$ \$ syntax forbids $W^{ \pm}$and $Z$ bosons to appear in $s$-channel propagators. Details of the approximation employed in MadGraph5_aMC@NLO for VBF- and VBS-type processes can be found in Ref. [44]. In this study we will consider matching to the shower Monte Carlos (SMCs) PYTHIA 8.230 [45] and HERWIG 7.1.2 [46] compiled with ThePEG 2.1.2. 


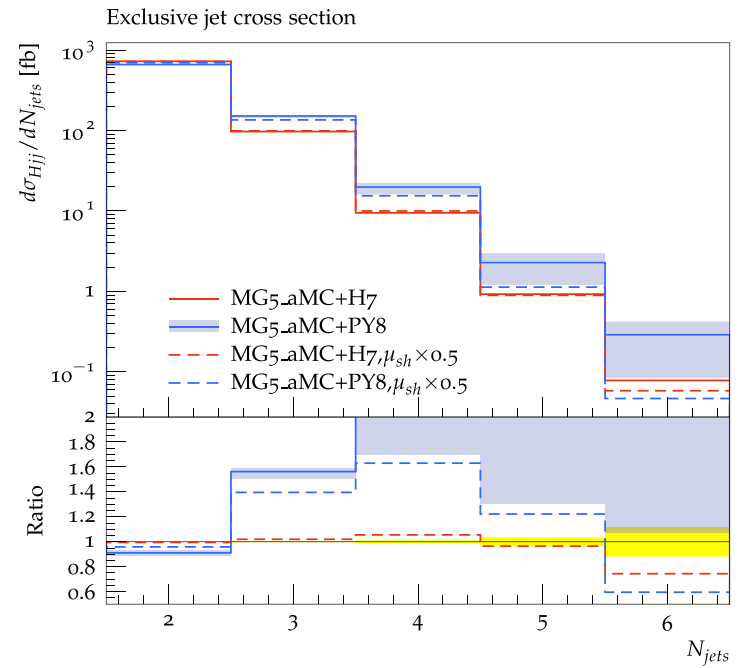

Fig. 2 Predictions at NLO + PS accuracy for the exclusive jet multiplicities (left) and for the transverse momentum of the Higgs boson (right) obtained with MadGraph5_aMC@NLO. Red and blue histograms correspond respectively to matching with HERWIG7 and PYTHIA8. Solid lines correspond to the default shower scale, while

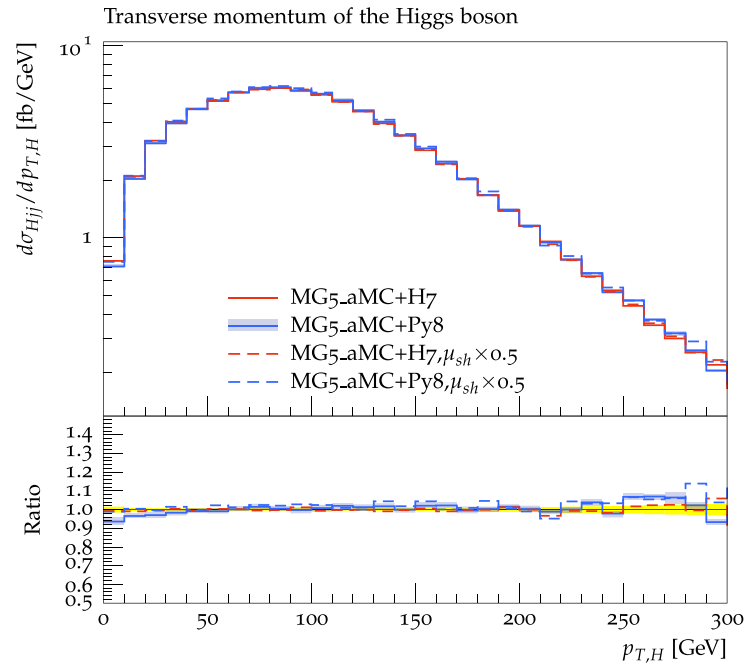

dashed ones correspond to a reduction of the default shower scale by a factor of two. For the PYTHIA8 prediction with default shower scale, the blue band illustrates the renormalisation and factorisation scale dependencies. Statistical uncertainties are not displayed for better readability in this and all subsequent plots
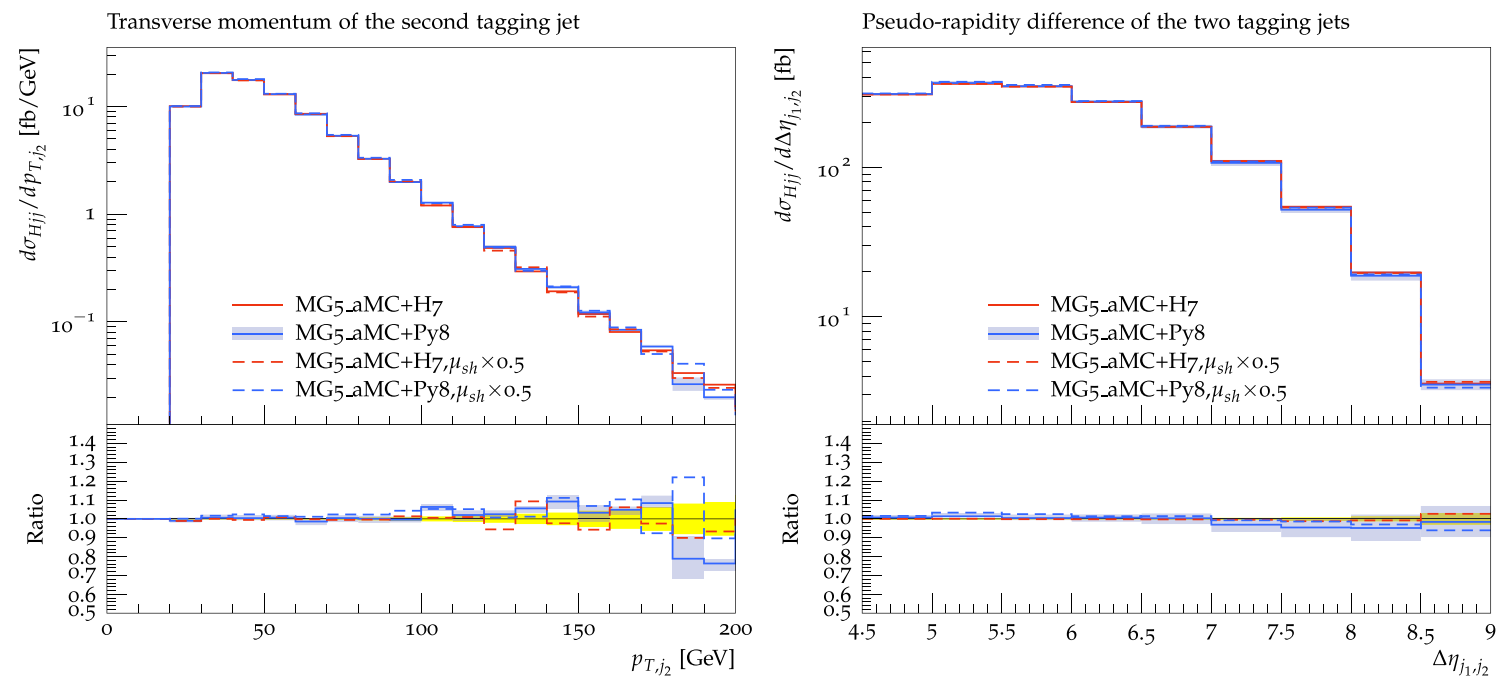

Fig. 3 Same as in Fig. 2, for the transverse momentum of the second tagging jet (left) and for the rapidity separation of the two tagging jets

\subsection{POWHEG-BOX}

The POWHEG-BOX [47] is a general framework for the matching of NLO calculations with parton shower programs making use of the POWHEG matching formalism [48,49]. Process-specific components have to be provided on a caseby-case basis. Higgs-boson production via VBF in association with two jets was one of the first processes being implemented in the POWHEG-BOX [8]. More recently, also code for VBF-induced Higgs production in association with three hard jets has been provided [18] being based on the matrix elements of Ref. [50] extracted from the VBFNLO code $[23,24]$. Both of these implementations rely on the VBF approximation. In this study we will consider matching to the SMCs PYTHIA 8.240 and HERWIG 7.1.4 compiled with ThePEG 2.1.4.

\section{3 proVBFH}

proVBFH v1.1.2 [13] is a public parton-level Monte Carlo program for the calculation of differential distributions for VBF Higgs boson production to NNLO-QCD accuracy in 


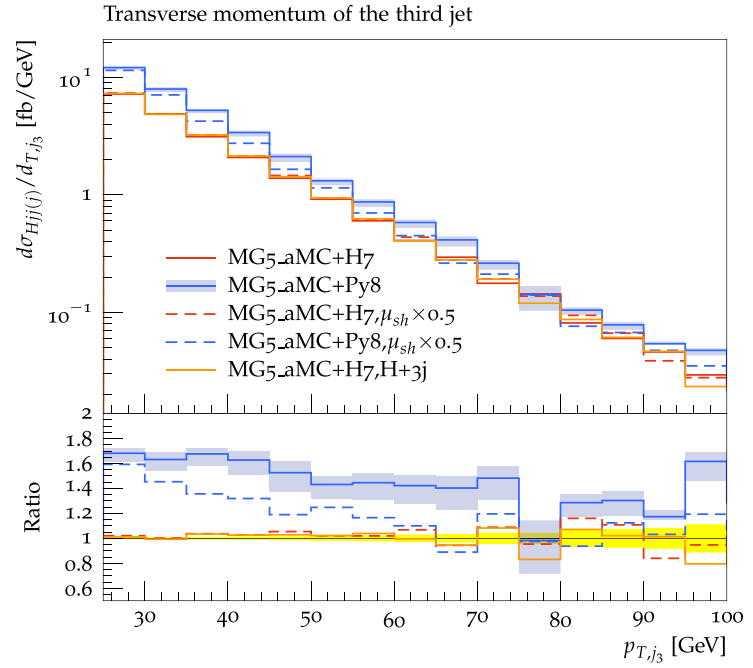

Fig. 4 Transverse momentum distribution of the third jet at NLO + PS accuracy as obtained with MadGraph5_aMC@NLO. Left: predictions for the production of a Higgs boson plus two jets via VBF, with the same colour-code as Fig. 2, together with the prediction for Higgs plus three jets via VBF matched with HERWIG7 (orange). Right: predictions for the production of a Higgs boson plus three jets via VBF, matched

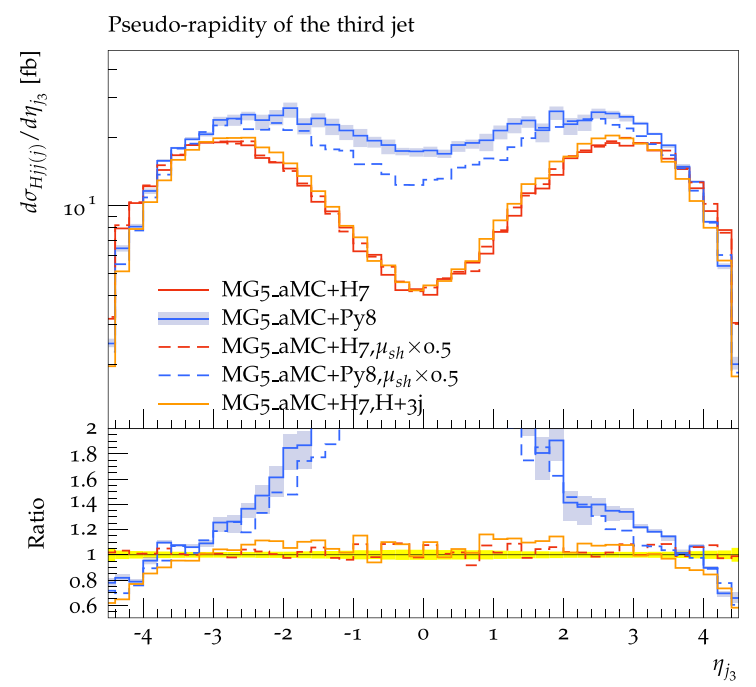

Fig. 5 Same as in Fig. 4, for the rapidity of the third jet

the VBF approximation. It is based on POWHEG's fully differential NLO-QCD calculation for Higgs boson production in association with three jets via VBF $[18,50]$, and an inclusive NNLO-QCD calculation [11], the latter being taken in the structure-function approximation. It achieves differential NNLO-QCD predictions through the projection-toBorn method introduced in [13]. proVBFH includes width effects for the internal $W$ and $Z$ bosons, and neglects fermion masses.

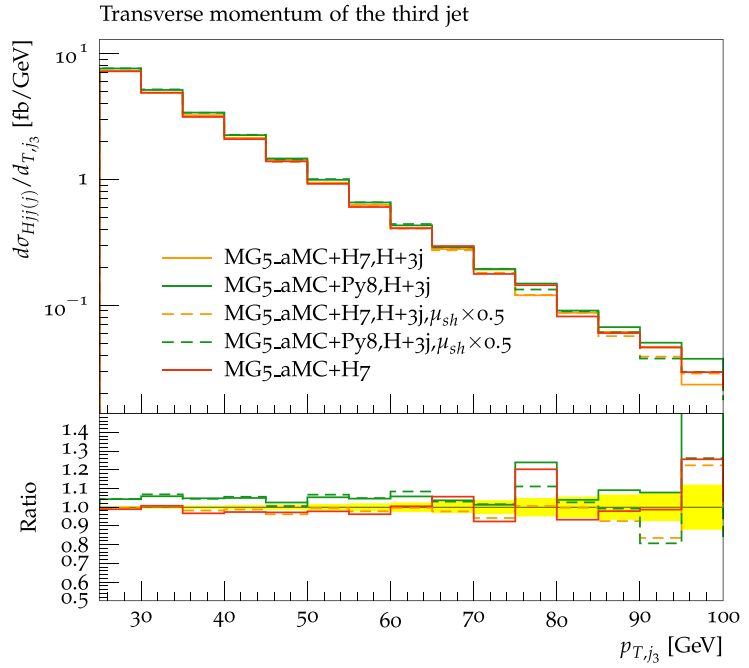

with HERWIG7 (orange) or PYTHIA8 (green), with nominal (solid) or halved (dashed) shower scale. In the same plot, the prediction for Higgs plus two jets via VBF matched with HERWIG7 (red solid) is shown. For the PYTHIA8 prediction for Higgs plus two jets via VBF with default shower scale, a blue band shows the renormalisation and factorisation scale dependence

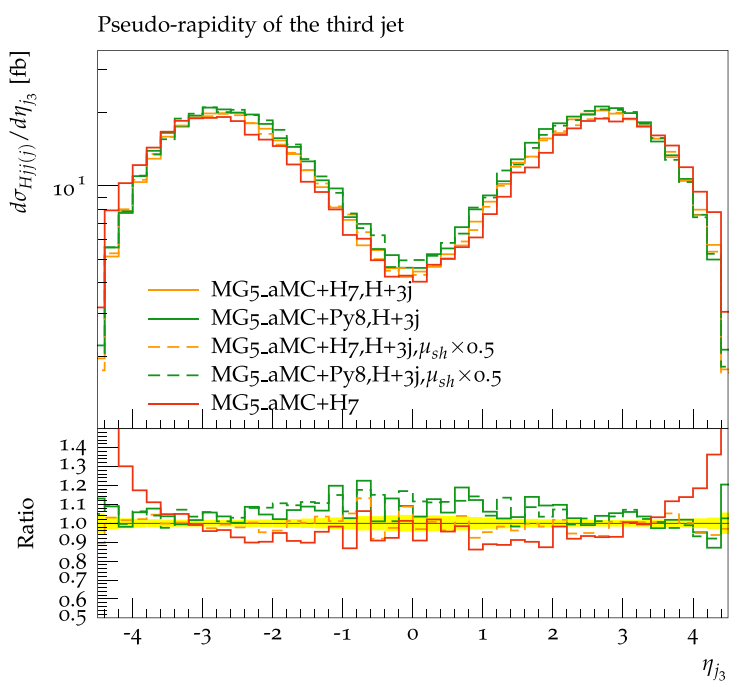

\subsection{VBFNLO and HJets + Herwig7/Matchbox}

The HERWIG7 event generator [21,46,51] features as one of its core components the Matchbox module [9], which can automatically assemble fixed-order and parton shower matched calculations with both the angular ordered [52] and dipole shower algorithms [53], using input from plugins providing matrix elements. The VBFNLO program $[23,54]$ is interfaced as one such module, providing NLO-QCD corrections to the $H j j$ and $H j j j$ production processes in the 


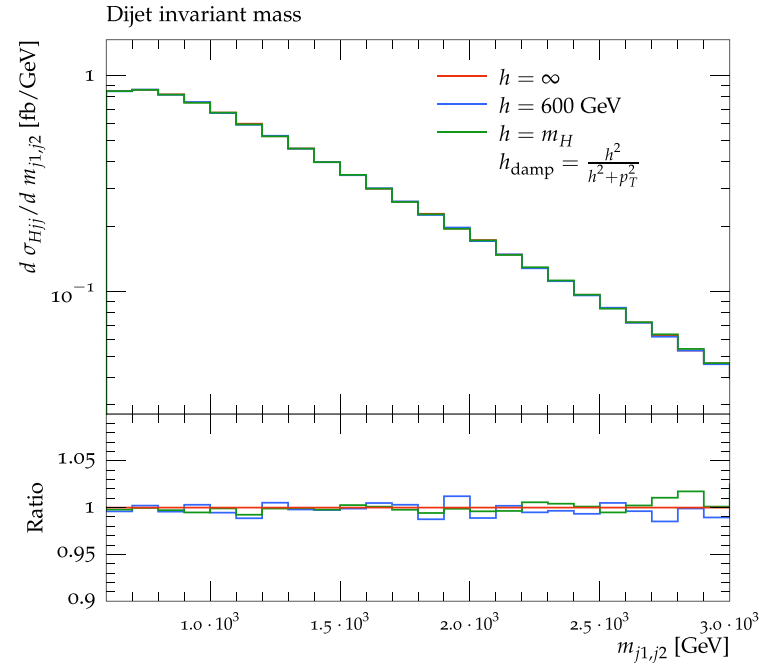

Fig. 6 Invariant-mass distribution of the two tagging jets (left) and transverse-momentum distribution of the third jet (right) within the cuts of Eqs. (3)-(4) at NLO + PS accuracy for the POWHEG-BOX, matched

VBF approximation. The HJets library [25] is an alternative module, providing matrix elements and NLO-QCD corrections for the full electroweak $H j j$ and $H j j j$ production processes without resorting to the VBF approximation.

In this study we consider the matching using the subtractive matching paradigm; a hard veto scale is imposed on the shower evolution to cut off the parton shower resummation at high transverse momenta. Its central value should reflect the hard transverse momenta at the process of interest, such that the shower evolution will not produce jets with significantly harder transverse momenta. A smearing is applied to the cutoff function, which we choose to be the "resummation" profile studied in more detail in Refs. [55,56]. Shower uncertainties are evaluated by varying the hard veto scale, which should reflect the bulk of the uncertainty both in the soft region and in regions which will be improved through the NLO matching.

\subsection{Recoil schemes in PYTHIA8}

By default PYTHIA8 employs a global recoil scheme for the generation of initial-state radiation. While this is certainly a valid approach when the underlying hard scattering does not have a colour flow between initial and final states, e.g. for colour-singlet production, it leads to inconsistencies when considering, for instance, Deep Inelastic Scattering (DIS), where the colour flow is only between an initial-state quark and a final state quark. This was discussed in Ref. [57] and a new dipole approach was introduced for initial-state radiation to better describe processes with initial-final colour flow. Since VBF can essentially be viewed as a double-DIS process

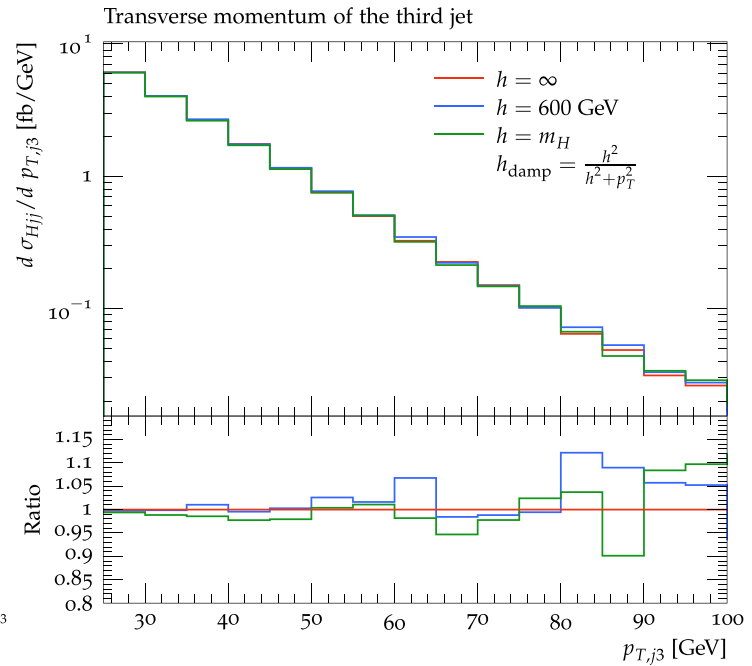

with PYTHIA8 using the dipole recoil scheme and considering hadronisation effects, for different choices of the hdamp parameter defined in Eq. (6)

where there is no QCD cross-talk between the two incoming protons, that discussion is also highly relevant here. It is known that in the VBF approximation a gluon emitted from one quark line cannot attach to the other quark line. It is therefore not very physical to distribute the recoil of such an emission over the entire event since such a prescription would destroy the relation between the kinematics and the soft radiation pattern. Instead one would expect the recoil to be along the quark line where the gluon emission took place. We therefore find it worth investigating the two different recoil schemes inside PYTHIA8 in this study. The dipole recoil scheme can be used directly with the POWHEG-BOX, whereas it is not currently possible with MadGraph5_aMC@NLO as the shower counterterms have been derived assuming a global recoil ${ }^{1}$. In the following we will therefore only show results using the dipole approach and the POWHEG-BOX. For the default (global) recoil scheme we show results obtained with both MadGraph5_aMC@NLO and the POWHEG-BOX. The inadequacy of a global-recoil scheme has been discussed for VBS processes in Ref. [44], and for $Z$-boson production via VBF in Ref. [59].

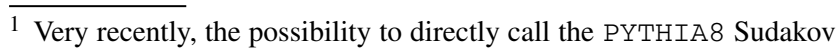
factor inside MadGraph5_aMC@NLO has been implemented [58]. Further developments in this direction may make it possible to also change parameters such as the recoil scheme and obtain the correct shower counterterm. We leave this for future work, possibly in collaboration with the authors of Ref. [58].
} 


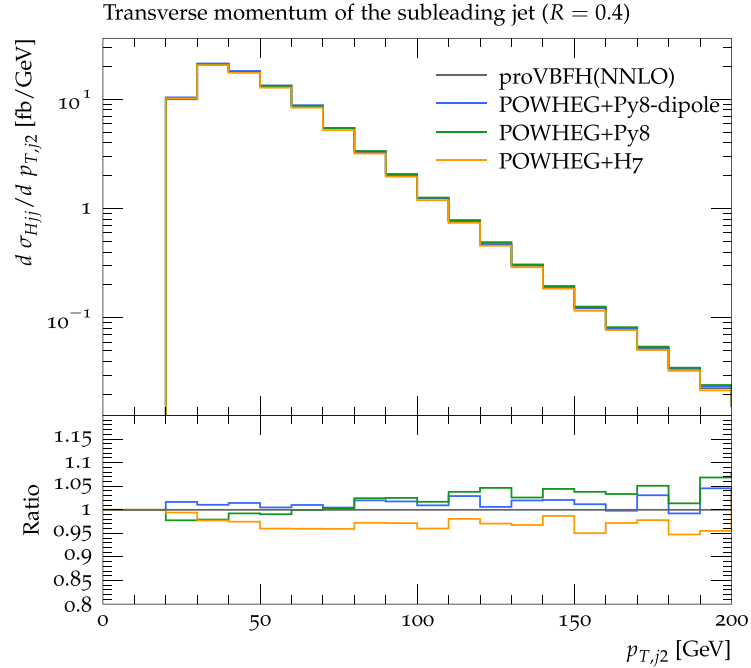

Fig. 7 Transverse-momentum of the second tagging jet (left) and separation of the two tagging jets in pseudorapidity (right) within the cuts of Eqs. (3)-(4) at NNLO, and at NLO + PS accuracy using the
Pseudo-rapidity difference of the two leading jets $(R=0.4)$

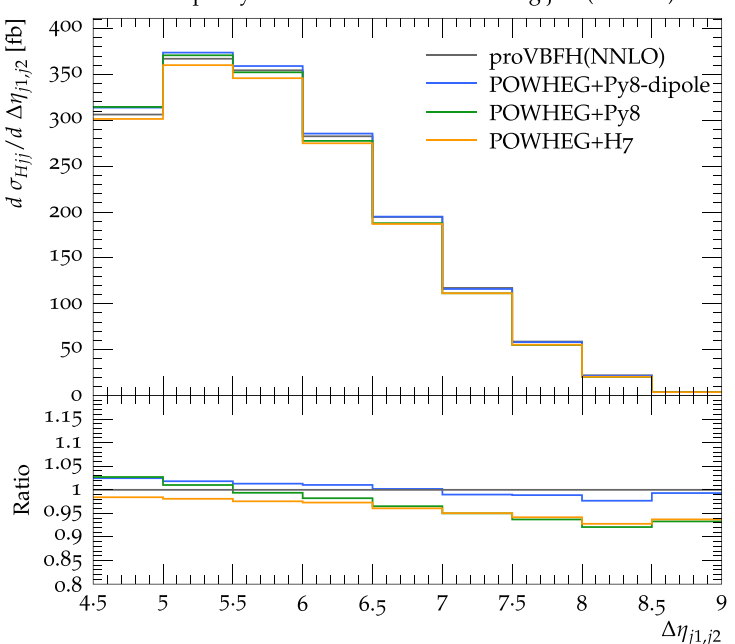

POWHEG-BOX matched with HERWIG7 and PYTHIA8 using two different recoil schemes. No hadronisation effects are taken into account

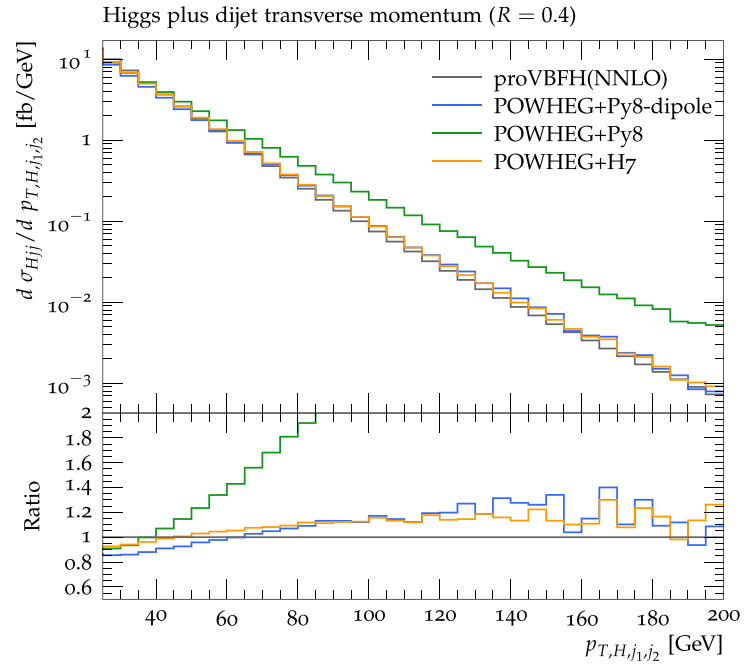

Fig. 8 Transverse-momentum of the Higgs-plus-tagging-jets system (left) and Zeppenfeld variable of the third jet (right) as defined in Eq. (7), within the cuts of Eqs. (3)-(4) at NNLO, and at NLO + PS accuracy

\section{Setup of the calculation}

\subsection{Input parameters}

We consider proton-proton scattering at the LHC with a centre-of-mass energy of $\sqrt{s}=13 \mathrm{TeV}$. For the PDFs of the proton we use an NNLO set with five massless flavours, PDF4LHC15_nnlo_100_pdfas [60], as provided by the LHAPDF 6 library [61] (identifier LHAPDF ID=91200) with the corresponding strong coupling, $\alpha_{\mathrm{S}}\left(M_{\mathrm{Z}}\right)=0.118$.

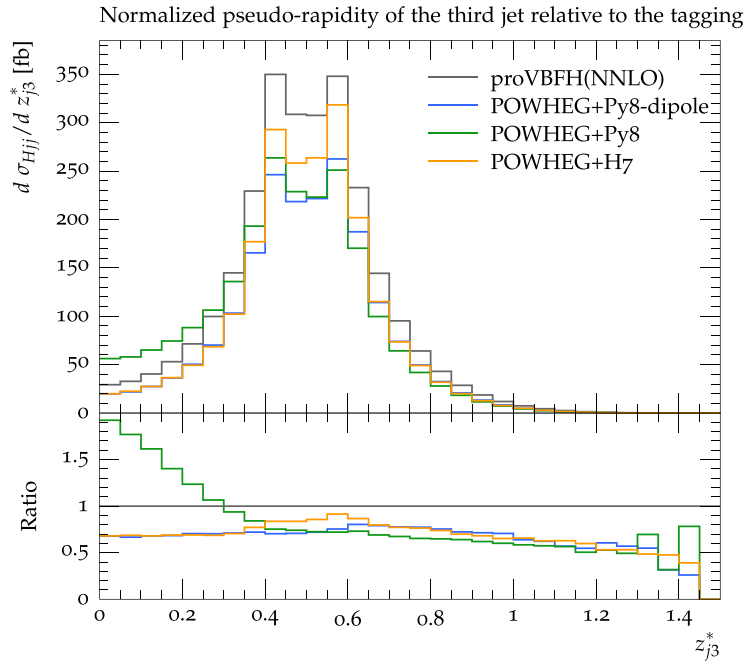

using the POWHEG-BOX matched with HERWIG7 and PYTHIA8 using two different recoil schemes. No hadronisation effects are taken into account

For the masses and widths of the particles entering our calculation the following values are used:

$$
\begin{aligned}
M_{\mathrm{Z}} & =91.1876 \mathrm{GeV}, & \Gamma_{\mathrm{Z}} & =2.4952 \mathrm{GeV}, \\
M_{\mathrm{W}} & =80.385 \mathrm{GeV}, & \Gamma_{\mathrm{W}} & =2.085 \mathrm{GeV}, \\
M_{\mathrm{H}} & =125.0 \mathrm{GeV} . & &
\end{aligned}
$$




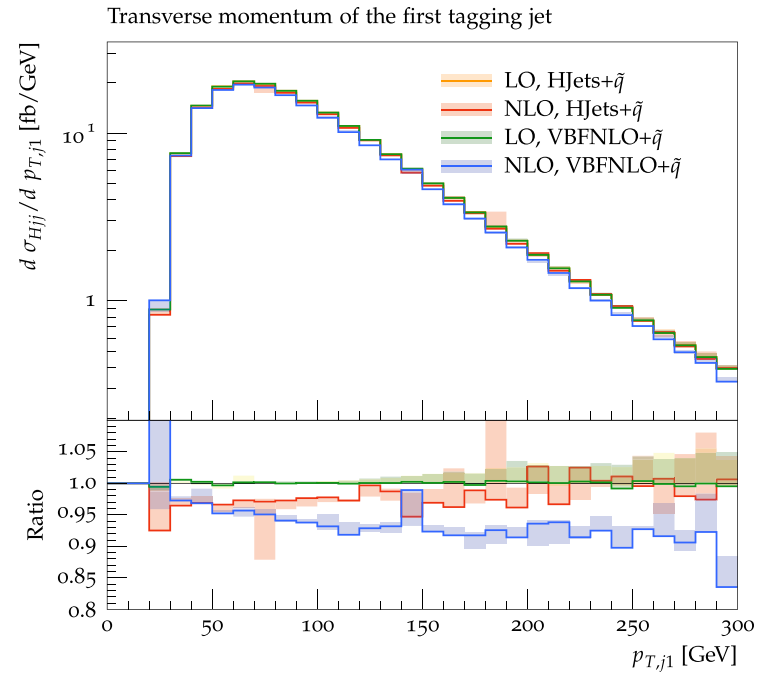

Fig. 9 Transverse-momentum distribution of the hardest jet (left) and the third jet (right) in the loose selection of Sect. 4.1.3, comparing HJets and VBFNLO with the angular ordered shower of HERWIG7.

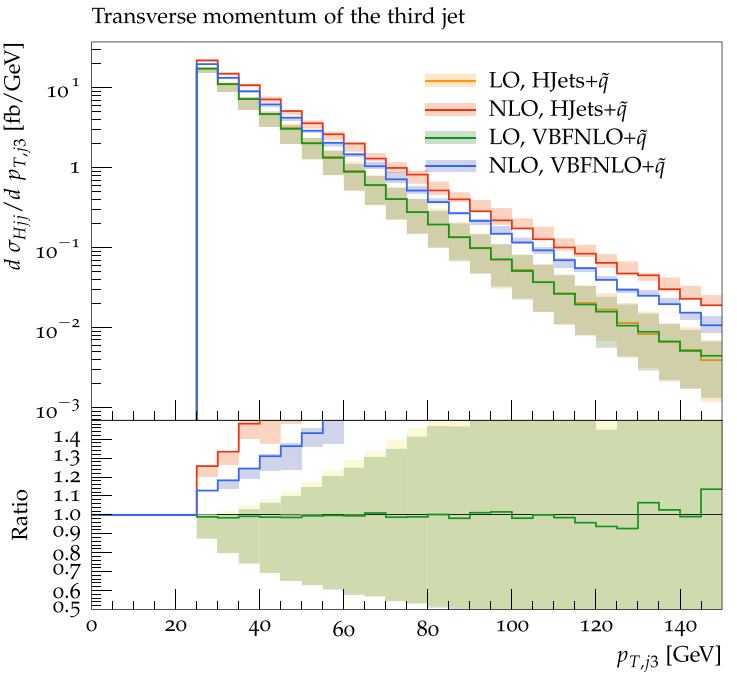

The coloured bands are obtained by varying the renormalisation and factorisation scales of the hard process by a factor of two around their central values
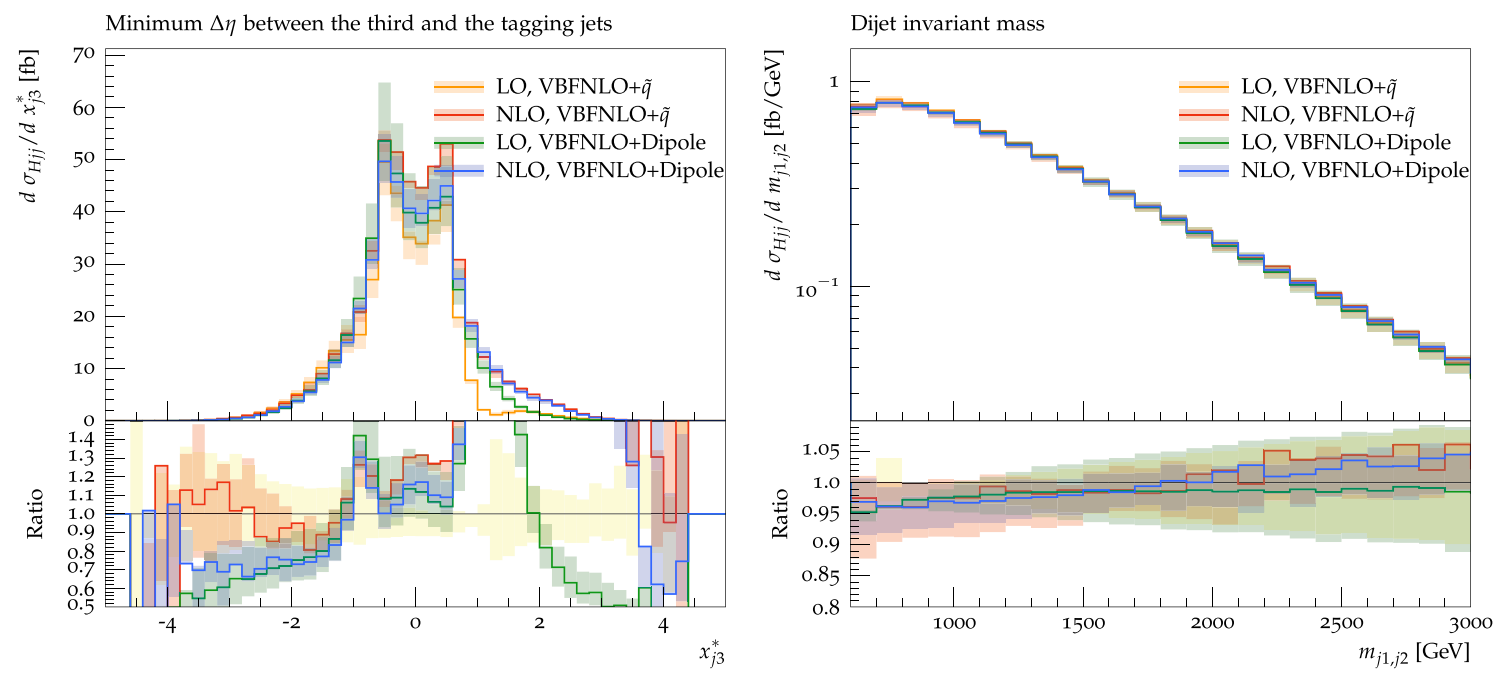

Fig. 10 Relative pseudo-rapidity difference between the third jet and the tagging jets (left) and tagging jet invariant mass (right). We use the setup of HERWIG7 + VBFNLO within the tight VBF selection of Sect. 4.1.3 and compare the dipole and angular ordered showers

As electroweak (EW) input parameters we use $M_{\mathrm{W}}, M_{\mathrm{Z}}$, and the Fermi constant, $G_{\mu}=1.16637 \times 10^{-5} \mathrm{GeV}^{-2}$. Other EW parameters, such as the EW coupling $\alpha$ and the weakmixing angle, are computed therefrom via tree-level EW relations. The Cabibbo-Kobayashi-Maskawa matrix is assumed to be diagonal, i.e. mixing effects between different quark generations are neglected.

The renormalisation scale, $\mu_{\text {ren }}$, and the factorisation scale, $\mu_{\text {fac }}$, are identified with $\xi_{\text {ren }} \mu_{0}$ and $\xi_{\text {fac }} \mu_{0}$, where the parameters $\xi_{\text {ren }}$ and $\xi_{\text {fac }}$ are to be varied between 1/2 and 2, and the central scale $\mu_{0}$, obtained from
$\mu_{0}^{2}=\frac{M_{\mathrm{H}}}{2} \sqrt{\left(\frac{M_{\mathrm{H}}}{2}\right)^{2}+p_{T, H}^{2}}$,

is computed from the mass and transverse momentum $p_{T, H}$ of the Higgs boson event by event. We do not include effects of hadronisation or underlying events in our simulations. 


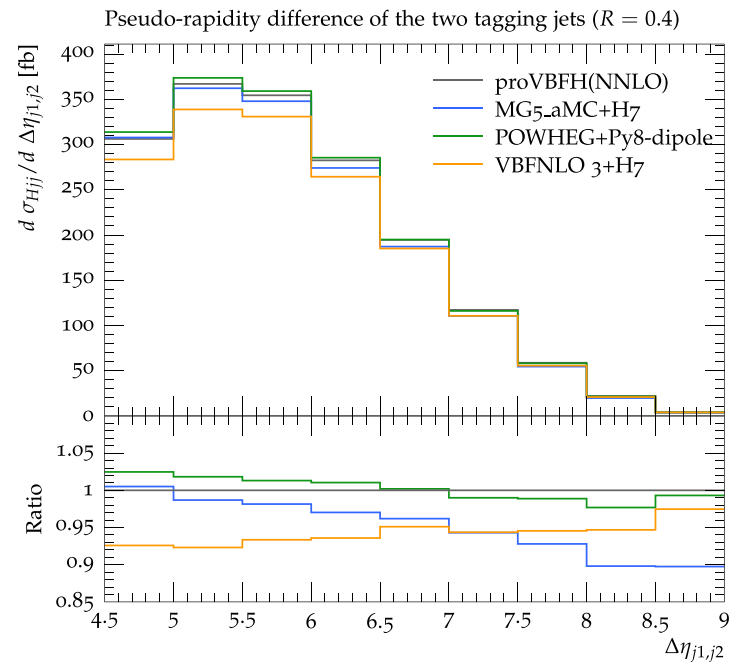

Fig. 11 Separation in pseudo-rapidity (left) and invariant-mass distribution of the two tagging jets (right) within the cuts of Eqs. (3)-(4) at NLO + PS accuracy for the MadGraph5_aMCaNLO, POWHEG-BOX, and VBFNLO+Herwig7/Matchbox gener-

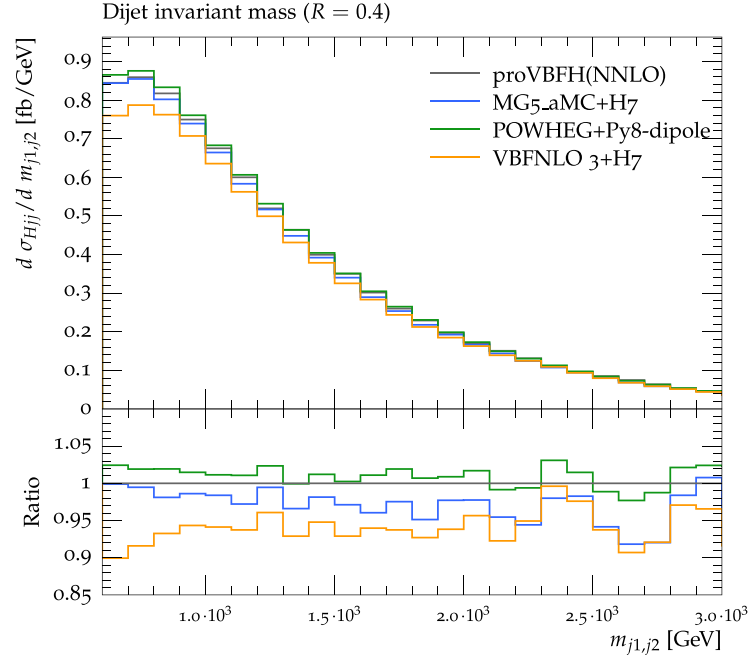

ators matched with HERWIG7 and PYTHIA8 using a dipole recoil scheme, respectively. Also shown are the NNLO-QCD predictions obtained with proVBFH

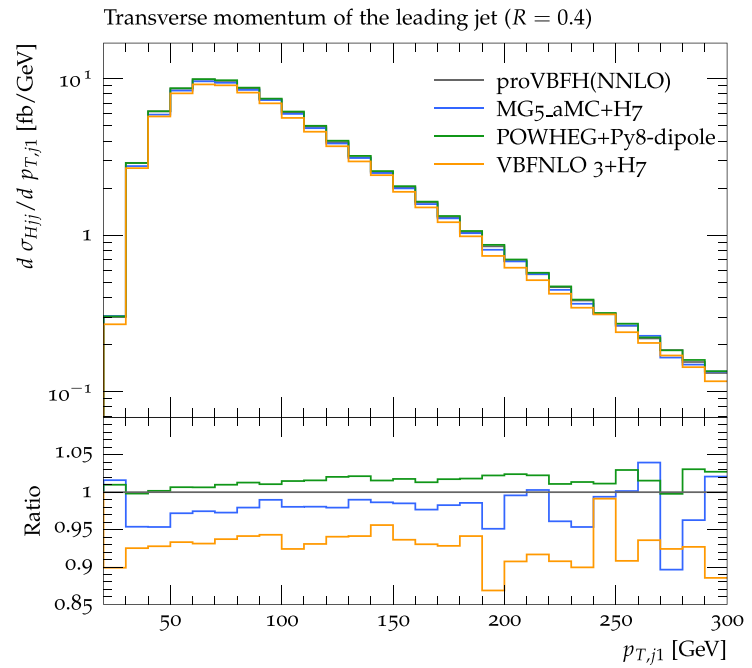

ators matched with HERWIG7 and PYTHIA8 using a dipole recoil scheme, respectively. Also shown are the NNLO-QCD predictions obtained with proVBFH

algorithm [62] using the Fast Jet package [63] with a distance parameter $R$. Unless specified otherwise, the value of $R$ is set to 0.4 . The thus produced jets need to exhibit a minimum transverse momentum and be located within the pseudo-rapidity range covered by the detector,

$p_{\mathrm{T}, \mathrm{j}}>25 \mathrm{GeV}, \quad\left|\eta_{\mathrm{j}}\right|<4.5$. 


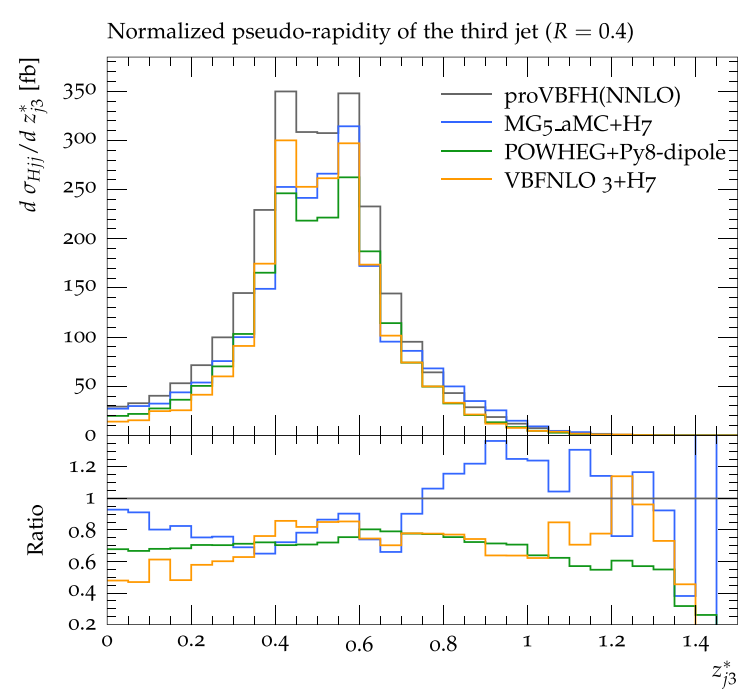

Fig. 13 Zeppenfeld variable of the third jet (left) and exclusive number of jets (right) within the cuts of Eqs. (3)-(4) at NLO + PS accuracy for the MadGraph5_aMC@NLO, POWHEG-BOX, and VBFNLO+Herwig7 / Matchbox generators matched with HERWIG7 and PYTHIA8

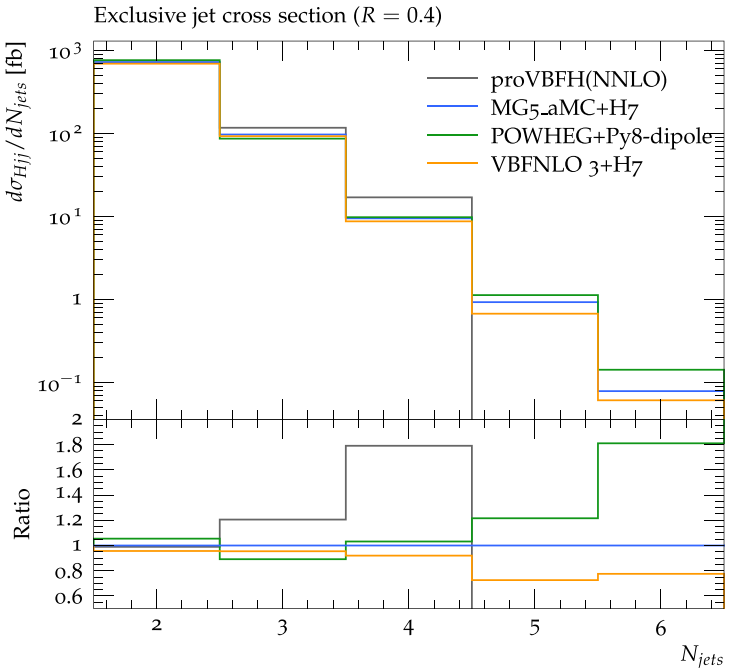

using using a dipole recoil scheme, respectively. Also shown are the NNLO-QCD predictions obtained with proVBFH. The ratio shown in the exclusive number of jets plots is taken with respect to the MadGraph5_aMC@NLO prediction
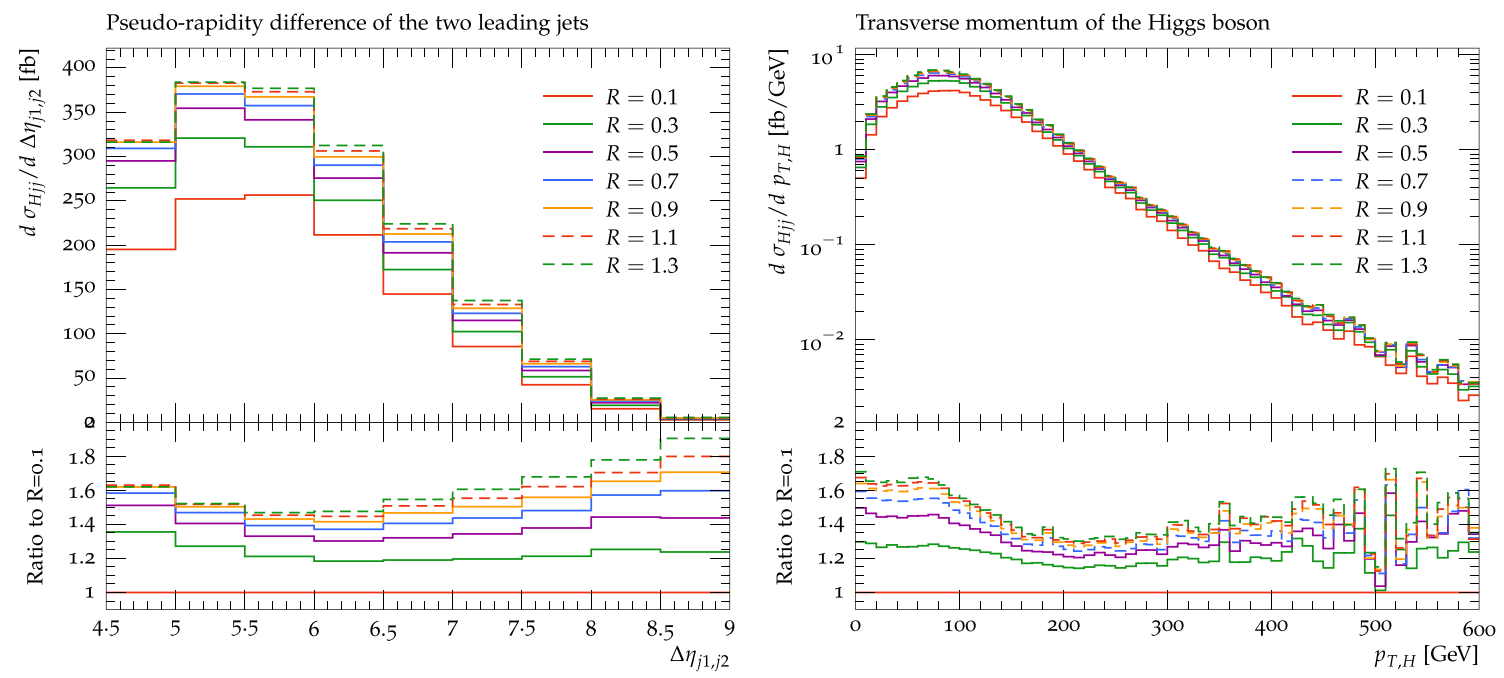

Fig. 14 The jet radius dependence illustrated for the pseudorapidity difference between the tagging jets, and the transverse momentum of the Higgs boson. Inclusive quantities also show a significant dependence on the jet radius due to selection criterion involving jets

The hardest two jets fulfilling this criterion are called "tagging jets". These two tagging jets are furthermore required to be located in opposite hemispheres of the detector, well separated in rapidity, and exhibit a significant invariant mass,

$\eta_{j_{1}} \cdot \eta_{j_{2}}<0, \quad\left|\Delta \eta_{\mathrm{j}_{1} \mathrm{j}_{2}}\right|>4.5, \quad m_{\mathrm{j}_{1} \mathrm{j}_{2}}>600 \mathrm{GeV}$.

\section{Numerical analysis}

In the following we will present the numerical results of our study. We will first discuss uncertainties specific to the indi- vidual generators. In the second part of this section, we will compare representative predictions of the individual generators with each other.

\subsection{Discussion of generator-specific uncertainties}

\subsubsection{Results from MadGraph5_aMC@NLO}

We now discuss results for VBF obtained with MadGraph5_aMC@NLO, and elaborate on effects due to the specific SMC employed and to the shower starting scale, on top of the usual estimate of theoretical uncertainties from the varia- 
tion of the hard (renormalisation and factorisation) scales. As SMCs, we consider the angular-ordered HERWIG7 generator and PYTHIA 8 with a global-recoil scheme. Concerning the shower starting scale $Q_{\text {sh }}$, it assigns (on an event-by-event basis) the maximum hardness of the radiation that the shower can generate in terms of the specific evolution variable, and is computed from a reference shower scale $\mu_{\mathrm{sh}}$. In general, one has $Q_{\text {sh }}=\mu_{\text {sh }}$ for the so-called $\mathbb{H}$-events, while for the S-events $Q_{\text {sh }}$ is generated from a probability distribution of which $\mu_{\text {sh }}$ is the upper endpoint. ${ }^{2}$. In order to assess the sensitivity of VBF observables on the shower scale, we choose to present results where either $\mu_{\mathrm{sh}}$ is not changed from its default value, or where it is halved. ${ }^{3}$

All plots, except those depicting properties of the third jet, which will be presented later, have the following layout: four histograms are displayed, with predictions obtained using PYTHIA8 (HERWIG7) in blue (red). Solid (dashed) histograms correspond to the default (halved) reference shower scale. In the inset, we show the bin-by-bin ratio over the prediction matched to HERWIG7 with nominal shower scale. A blue band, corresponding to the hard-scale variations (the renormalisation and factorisation scales are varied independently by a factor of two around the central value giving rise to a nine-point variation) is displayed for the prediction matched to PYTHIA8 with the nominal shower scale.

The first observable we consider is the exclusive ${ }^{4}$ jet multiplicity, in the left panel of Fig. 2. When looking at this figure, one should bear in mind that the two-jet bin is the only bin with genuine NLO accuracy. The three-jet bin is only LO accurate, while higher multiplicities of jets are entirely due to the SMC. A consequence of this is the agreement among predictions in the two-jet bin, where predictions lie within $10 \%$ of each other, with those matched to PYTHIA8 predicting a lower rate than those with HERWIG7. In the three-jet bin, on the other hand, we observe large discrepancies, not covered by the hard-scale uncertainty: the predictions matched with PYTHIA8 exhibits a $60 \%$ excess with respect to the one matched with HERWIG7. Such a large effect is due to the global recoil scheme employed by PYTHIA8 in order to be consistent with the matching in MadGraph5_aMCanLO, which is not suitable for VBF/VBS-type processes, c.f. our discussion in Sect. 2.5. For higher-multiplicity bins discrepancies and scale uncertainties become huge. Finally,

\footnotetext{
${ }^{2}$ Details can be found in Sect. 2.4.4 of Ref. [19] and, for a processspecific example, in Sect. 3.2 of Ref. [64] In particular, for processes without light jets at the Born level one has $\mu_{\mathrm{sh}}=H_{T} / 2\left(H_{T}\right.$ being the total transverse energy of the event); in the case relevant for VBF, where there are $n$ jets already at Born level, $\mu_{\mathrm{sh}}=d_{n}-d_{n+1} / 2$, where $d_{i}$ is the $i$-th $k_{T}$ distance of the jets obtained by clustering the partons.

3 This can be done by setting the shower_scale_factor variable to 0.5 inside the run_card of MadGraph5_aMC@NLO.

${ }^{4}$ For this observable the bin corresponding to $n$ jets is filled when there are exactly $n$ jets in an event.
}

we remark that predictions matched with PYTHIA 8 display a more pronounced sensitivity on the shower starting scale, while for HERWIG7 such a dependence is very small.

The next observable we consider is the transverse momentum distribution of the Higgs boson, in the right panel of Fig. 2. This observable displays an excellent agreement among all predictions, with discrepancies of few percents at most, a behaviour which is common for observables inclusive in the number of jets: indeed, the differences in the two- and three-jet bins described before tend to compensate almost exactly. We have verified that this applies for many other NLO-accurate observables, such as those related to the first and second tagging jet. As representative ones, we show the transverse momentum of the second tagging jet and the rapidity separation of the two tagging jets in Fig. 3. We remark that the dependence on the renormalisation, factorisation, and shower scales for these observables is very small, with the exception of the rapidity separation at large rapidities, comparable to the differences among predictions employing the two parton showers.

We now turn to observables related to the third jet, in particular the transverse momentum and rapidity distributions, respectively, shown in Figs. 4 and 5. In order to reach NLO accuracy also for these observables, we additionally show predictions for the production of a Higgs boson in association with three jets via VBF at NLO + PS accuracy, both matched with HERWIG7 (orange) and with PYTHIA8 (green). The line pattern (solid or dashed) has the same meaning as above. For the sake of better readability, we show two panels for each observable. In the left (right) panel, we show the four predictions for the production of a Higgs boson plus two (three) jets via VBF and the one for the production of a Higgs boson plus three (two) jets matched with HERWIG7 using the default shower scale. In the inset we show the respective ratios over the prediction for the production of a Higgs boson plus two (three) jets via VBF, matched with HERWIG7 and with nominal shower scale. The plotting range is different in the inset of the left and right panels. The predictions for the rapidity distribution of the third jet for the production of a Higgs boson plus two jets via VBF at NLO + PS show that the origin of the excess observed in the jet multiplicities when matching to PYTHIA 8 mainly comes from jets in the central region, as a consequence of the globalrecoil scheme. The same effect is rather flat in the transverse momentum spectrum. It is worth to observe that reducing the shower scale is not sufficient to cure this behaviour, and that the renormalisation and factorisation scale variations fail to cover differences among the shower generators. Indeed, such a behaviour is unphysical, which can be understood by looking at the predictions for the production of a Higgs boson plus three jets at NLO + PS accuracy. The difference among various predictions is now reduced to the $10 \%$ level or below, thanks of the better perturbative description of these observ- 
ables. It is important to stress that the PYTHIA 8 predictions still employ a global-recoil scheme, in accordance with the needs of the matching in MadGraph5_aMC@NLO. It is also worth to notice the impact of the correction (in the case of HERWIG7) when passing from an LO description (Higgs plus two jets via VBF) to an NLO one (Higgs plus three jets via $\mathrm{VBF}$ ): while no visible effect can be appreciated in the transverse momentum spectrum, looking at the rapidity one can see how the NLO corrections tend to enhance central rapidities and deplete larger ones $(|\eta|>3.5)$.

In conclusion, supported by the results presented in this section and given the impossibility to employ PYTHIA 8 in conjunction with a dipole-recoil scheme within MadGraph5_aMC@NLO, we strongly advise to use MadGraph5_aMC@NLO only in conjunction with HERWIG7 for the simulation of VBF.

\subsubsection{Results of the POWHEG-BOX}

In the POWHEG-BOX an assessment of the intrinsic uncertainty related to the POWHEG matching procedure is possible by a variation of the so-called hdamp parameter. This parameter governs the splitting of the full real-emission contribution $R$ into a singular part, $R_{S}$, that enters into the Sudakov form factor and a regular part, $R_{f}$, according to

$R_{s}=R \times$ hdamp,

$R_{f}=R \times(1-$ hdamp $)$,

with

hdamp $=\frac{h^{2}}{h^{2}+p_{T}^{2}}$,

where $p_{T}$ denotes the transverse momentum of the hardest parton of the real-emission contribution and $h$ is a parameter that can be set by the user. We explore the matching uncertainty accessible via the hdamp parameter by considering the three cases $h=\infty$ (i.e. no damping), $h=M_{\mathrm{H}}$, and $h=m_{\mathrm{j}_{1} \mathrm{j}_{2}}^{\min }=600 \mathrm{GeV}$. We show plots using PYTHIA8 as the SMC with the dipole recoil strategy [57].

Naively, one would expect observables related to the hard jets that are not very sensitive to soft emission to be less affected by the choice of the hdamp parameter than distributions related to the sub-leading jets. To assess this expectation, in Fig. 6 we show examples of both types of observables in the VBF $H j j$ process. The invariant-mass distribution of the two tagging jets is completely insensitive to the value of $h$. However, the same holds true also for the transversemomentum distribution of the third jet over the entire range considered, where larger effects might be expected. This finding clearly indicates that the VBF process considered here is quite insensitive to the actual form of the Sudakov form factor used for the POWHEG-BOX simulation. We remark that, consequently, the choice of the hdamp parameter has little impact on the numerical stability and CPU requirements of the program. We will therefore use the value $h=\infty$ (corresponding to hdamp $=1$ ) as a default.

While the dependence of predictions obtained with the POWHEG-BOX on hdamp obviously is very small, another source of generator-specific uncertainty is constituted by the choice and settings of the SMC, the POWHEG-BOX is matched to. To explore this effect we present a systematic comparison of NLO + PS predictions obtained with PYTHIA8 (both default and dipole recoil scheme, c.f. Sect. 2.5), angular ordered HERWIG7, and fixed-order results at NNLO-QCD accuracy obtained with the proVBFH program. We expect only a small impact of the SMC choice on observables with little sensitivity to soft radiation effects, such as the transverse momenta of the tagging jets and related distributions. Indeed, as illustrated by Fig. 7, the transversemomentum distribution of the second tagging jet is very stable with respect to the choice of SMC, and indeed the NLO + PS simulation provides a very good approximation for the NNLO prediction. Small differences are also observed in the rapidity separation of the two tagging jets, shown in the righthand-side of Fig. 7. We notice, however, that in this case the results obtained with the dipole recoil scheme in PYTHIA8 lie clearly above the HERWIG7 results, while the default version of PYTHIA 8 resembles the HERWIG7 predictions in the region of highly separated jets, but reproduces the PYTHIA8 results in the dipole scheme for smaller rapidity separations.

Much more pronounced differences between the various SMC choices are found for distributions related to the subleading jets. Figure 8 shows the transverse-momentum distribution of the system formed by the Higgs boson and the two tagging jets, which reflects the transverse momentum of the remaining objects produced in the scattering process, in particular the non-tagging jets. Since such subleading jets in the $H j j$ simulation can only be accounted for by the realemission matrix elements or parton-shower emission they are only described at leading order or parton-shower accuracy. In the tail of the $p_{T, H, j_{1}, j_{2}}$ distribution, the PYTHIA 8 default results by far exceed the reference results constituted by the NNLO prediction, while no such large differences are observed in the HERWIG7 and PYTHIA 8 results using the dipole recoil scheme.

A variable particularly suitable to indicate the relative position of the third jet with respect to the centre of the tagging-jet system is constituted by the so-called Zeppenfeld variable, defined as

$z_{\mathrm{j}_{3}}^{\star}=\frac{\eta_{\mathrm{j}_{3}}-\frac{\eta_{\mathrm{j}_{1}}+\eta_{\mathrm{j}_{2}}}{2}}{\left|\Delta \eta_{\mathrm{j}_{1} \mathrm{j}_{2}}\right|}$

For small values of $z_{j_{3}}$ the third jet is right in between the two tagging jets, while larger $z_{\mathrm{j}_{3}}$ values correspond to more 
peripheral configurations. The $z_{j_{3}}^{\star}$ distribution helps to understand where the large differences between the various SMC simulations stem from. Obviously, the PYTHIA8 default scheme produces an abundance of radiation for small values of $z_{j_{3}}^{\star}$, i.e. in between the two tagging jets.

\subsubsection{VBFNLO and HJets + Herwig7/Matchbox}

Within the setup using the HERWIG7 interface to VBFNLO and HJets we perform the subtractive, MC@NLO-type matching and assess the uncertainties by varying the hard scale of the shower evolution as well as the factorisation and renormalisation scales of the hard process. For a detailed discussion of these uncertainties see [55,65], where VBS processes have been considered as well. We also investigate the difference between the default, angular ordered $\tilde{q}$ shower, as well as the dipole-type evolution which is available as an alternative module. Since the HJets module [25] implements the calculation without any VBF approximation, we can perform a comparison to VBFNLO, which resorts to the VBF approximation that is also used in the POWHEG-BOX and MadGraph5_aMC@NLO generators. We find quite similar results of the showering in between the two HERWIG7 shower modules, as well as similar variations and stability with respect to the fixed order input.

We first compare the VBF approximation for both a tight and a loose cut setup with subsequent parton showering, including the variations from the renormalisation and factorisation scales. The tight setup is defined by the cuts of Sect. 3.2, while for the loose setup we relax the selection to

$\left|\Delta \eta_{j_{1} j_{2}}\right|>1, \quad m_{j_{1} j_{2}}>200 \mathrm{GeV}$,

with all other cuts identical to the general setup. Examples are depicted in Fig. 9, where we generally find a large discrepancy between VBFNLO and HJets for the third jet in a loose setup, and a very good agreement in between the two for a tight VBF selection. Similar findings at fixed order also apply to the third jet distributions, see [66]. Within a tight VBF selection, the shower uncertainties in the NLO matched case are at the few-percent level for observables involving the hardest three jets, but can still be significant for higher jet multiplicities, something which we exemplify in Fig. 10, where we include the minimum rapidity difference of the third jet with respect to the tagging jets, defined by

$x_{j_{3}}^{*}=\min \left\{\left|\eta_{j_{1}}-\eta_{j_{3}}\right|,\left|\eta_{j_{2}}-\eta_{j_{3}}\right|\right\}$,

where $x_{j_{3}}^{*}$ receives a minus sign if the third jet is outside the dijet window, i.e. if $z_{j_{3}}^{\star}>0.5$. We also show the dijet invariant mass distribution.

\subsection{Comparison of different generators}

Having investigated variations within the individual SMCs we now turn to a study of the three generators in the recommended default setup. A summary of the setups used with the three different generators is given in Table 1. Given the above discussion we show results for MadGraph5_aMC@NLO interfaced to HERWIG7, the POWHEG-BOX interfaced to PYTHIA8 using the dipole recoil strategy, and VBFNLO+Herwig7/Matchbox. All three generators use the VBF approximation, and have been checked to agree within statistical uncertainties when run at fixed-order (at the inclusive and differential level). Hence we expect any disagreement to arise only from differences in matching procedure and shower details rather than the fixed-order matrix elements for the hard scattering. We recall that we do not include hadronisation or underlying event effects in the comparison.

In Fig. 11 we show the typical VBF observables; tagging jet rapidity separation, $\Delta \eta_{j_{1}, j_{2}}$, and invariant mass, $m_{j_{1}, j_{2}}$, for MadGraph5_aMC@NLO (blue), POWHEG-BOX (green), and VBFNLO+Herwig7 / Matchbox (orange). We also show the fixed order NNLO-QCD prediction obtained using proVBFH (black). The plot shows a spread in predictions of less than $10 \%$. Both POWHEG-BOX and MadGraph5_aMC@NLO show the same shape distortion with respect to proVBFH although they have different normalisation. VBFNLO+Herwig7 / Matchbox, on the other hand, exhibits a different slope behaviour in both observables with respect to the other two generators.

There are also some differences between the three generators when considering more inclusive observables. However in this case the discrepancies are mostly due to differences in normalisations. To illustrate that point, in Fig. 12 we show the transverse momentum of the Higgs boson and of the first tagging jet in the event. All three generators agree within $10 \%$ and have very similar shapes. In particular, all three generators are comparable in shape with respect to the fixed order NNLO-QCD prediction.

Lastly we show a comparison of the Zeppenfeld variable $z_{\mathrm{j}_{3}}^{\star}$ and the exclusive jet multiplicity in Fig. 13. We remind the reader that all three considered generators have LO accuracy for three-jet observables and pure shower accuracy for observables with more than three jets. Although there are larger differences between the generators for $z_{\mathrm{j}_{3}}^{\star}$, of the order of 20\%, they have fairly similar shapes up to about $z_{j_{3}}^{\star} \lesssim 0.8$ and, in particular, none of the predictions exhibits a large excess in the small $z_{j_{3}}^{\star}$ region. For the exclusive jet cross section it is clear that matched calculations predict a much smaller number of jets than the fixed order prediction in the three and four jet bins. They do, however, agree amongst each other at the $10 \%$ level for the 2, 3 and 4 jet rates. The discrepancy with respect to the fixed order prediction is related 
Table 1 The various generators used in the comparisons throughout this paper and their respective settings

\begin{tabular}{|c|c|c|c|c|}
\hline Generator & Matching & SMC & Shower recoil & Used in Sect. 4.2 \\
\hline VBFNLO+Herwig7 /Matchbox & $\oplus$ & HERWIG 7.1.5 & Global $(\tilde{q}) /$ local (dipole) & $\checkmark(\tilde{q})$ \\
\hline HJets + Herwig7/Matchbox & $\oplus$ & HERWIG 7.1.5 & Global $(\tilde{q}) /$ local (dipole) & \\
\hline MadGraph5_aMC@NLO 2.6.1 & $\oplus$ & HERWIG 7.1.2 & Global & $\checkmark$ \\
\hline MadGraph5_aMC@NLO 2.6.1 & $\oplus$ & PYTHIA 8.230 & Global & \\
\hline POWHEG BOX V2 & $\otimes$ & PYTHIA 8.240 & Local (dipole) & $\checkmark$ \\
\hline POWHEG BOX V2 & $\otimes$ & PYTHIA 8.240 & Global & \\
\hline POWHEG BOX V2 & $\otimes$ & HERWIG 7.1.4 & Global $(\tilde{q})$ & \\
\hline
\end{tabular}

The column 'matching' refers to either MC@NLO $(\oplus)$ or POWHEG $(\otimes)$ style matching. For a more detailed discussion of the setup of the various generators please see Sects. 4.1.1-4.1.3. The last column indicates which setup is being used in the final comparison of Sect. 4.2

to soft radiation produced by the shower that is lost outside of the rather narrow jet cone.

\subsection{Jet radius dependence}

In this section we consider the dependence of the VBF cross sections on the jet radius $R$ after showering, but without any hadronisation or underlying event for which we expect a parametrically different dependence on the jet radius. From parton showering, and higher order corrections in general, we expect a leading $\log (1 / R)$ dependence, which has previously been studied for VBF processes in Ref. [67], and for more general processes involving hard jets the interplay with scale choices and variations at fixed order has also been investigated [68]. We show some of the results in Fig. 14. While we have not attempted to perform any fit of the $R$ dependence, the general pattern we see is that after parton showering leading, as well as next-to-leading order matched predictions show a similar, and significant $R$ dependence. This dependence does not only affect the normalisation of the cross section due to the jet selection criteria, but also the shapes even for inclusive distributions like the Higgs boson transverse momentum. A comprehensive discussion of the jet radius dependence needs not only to include a study of the behaviour of NLO QCD corrections, but also to include the impact of hadronisation and multi-parton interactions. Preliminary results for investigating the jet radius dependence at NNLO have also been reported in Ref. [69].

\section{Recommendations and conclusions}

In this work we performed a quantitative investigation of parton-shower and matching uncertainties of perturbative origin for the production of a Higgs boson plus two jets via VBF. The relevance of such a study is supported by the fact that, already in analyses based only on part of the data taken during Run II of the LHC, for VBF the dominant source of uncertainties are theoretical ones. Improving on Higgs anal- yses in the VBF channel thus crucially requires a quantitative understanding of the tools used for the simulation of Higgs production via VBF.

In the study of matching uncertainties, we found that, within a single generator and SMC, theoretical uncertainties estimated by the usual renormalisation and factorisation scale variations, possibly supplemented by variations in a variable that controls the shower hardness (shower starting scale for MadGraph5_aMC@NLO or hdamp for the POWHEG-BOX), turn out to be small, hardly above the fewpercents figure. This also applies to the hard shower scales variations in HERWIG7, which can become more significant if properties of the third jet are probed. However we showed that the differences among predictions obtained with different SMCs can be more significant, easily exceeding the aforementioned estimate of theory uncertainties. For observables described at NLO-QCD accuracy, these differences are at the $10 \%$ level. However, they are mostly due to normalisation effects, while shapes of distributions are described to an even better accuracy when the various NLO + PS programs and the NNLO result are compared. For LO-accurate observables, differences turn out to be much larger, but not always physical. A prominent example is the description of third-jet observables when PYTHIA8 is employed with a global-recoil scheme, which gives a huge enhancement in the central-rapidity region. Such an enhancement has been proven to be unphysical by looking at an NLO-accurate description of the same variable, where it disappears. Taking this fact into account, uncertainties for third-jet observables can be quantified in the $20 \%$ domain.

As a consequence, we recommend against using PYTHIA 8 with a global-recoil scheme for VBF, in a simulation based on Higgs plus two jet production at $\mathrm{NLO}^{5}$. Instead one should change the recoil scheme to the dipole one when this is com-

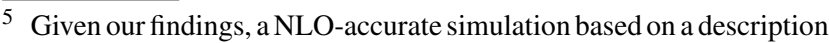
with three additional jets for the relevant observables, or on the merging of different multiplicities, should still provide sensible results even with PYTHIA8 and a global recoil.
} 
patible with the matching (i.e. with POWHEG-BOX). When this is not (yet) the case (with MadGraph5_aMC@NLO) one should use an entirely different SMC like HERWIG7, which performs the matching internally and uses recoil schemes which respect the colour flow information of the hard process either through the initial conditions to the angular ordered evolution in case of the default $\tilde{q}$-shower or the nature of the alternative dipole shower algorithm, which lead to comparable results.

We conclude that, within the typical VBF phase space, all the programs considered in this study yield reliable results. However, we remind the reader that because of the VBF approximation used in most of the considered generators, valid predictions can only be expected after appropriate selection cuts are employed. As far as VBF Higgs processes are concerned, the HJets plugin to HERWIG7 can provide accurate predictions for $H+2$ jet and $H+3$ jet final states at NLO QCD without resorting to the VBF approximation and we have used this as an explicit check to demonstrate good agreement within the VBF selection region.

We also stress that a comprehensive study of uncertainties for VBF predictions necessarily needs to include the effects of multi-parton interactions, colour reconnection and hadronisation. The impact of these effects will vary largely with the jet radius and need to be confronted with the perturbative variations in order to obtain a global picture. We leave such a study to future work. It is important to stress that the impact of including these effects should not be mistaken for the size of uncertainty induced thereby. Instead, a careful evaluation of the uncertainties associated with these effects is required, specifically in response to perturbative variations and a (re)tuning cross-check.

We have included the RIVET [70] analysis used in this study with the ancillary files of the arXiv submission for anyone interested in reproducing our results.

Acknowledgements This work has received support in part by the COST actions CA16201 "PARTICLEFACE" and CA16108 "VBSCAN" The authors would also like to thank the LHC Higgs Cross Section Working Group for stimulating discussions and Michael Rauch for contributions in the early stages of this work. A. K. would like to thank Frédéric Dreyer and Gavin Salam for useful comments on the manuscript. M.Z. would like to thank Rikkert Frederix for clarifications on the shower scale, and all the authors of MadGraph5_aMC@NLO for various discussions. The work of B. J. and J. S. has been supported in part by the German Federal Ministry for Education and Research (BMBF) under grant number 05H18VTCA1. B. J. and J. S. furthermore acknowledge support by the state of Baden-Württemberg through bwHPC and the German Research Foundation (DFG) through Grant no INST 39/963-1 FUGG. A. K. is supported by the European Research Council (ERC) under the European Union's Horizon 2020 research and innovation programme (Grant agreement No. 788223, PanScales), and by Linacre College, Oxford. The work of S. P. was supported in part by the European Union's Horizon 2020 research and innovation programme as part of the Marie Skłodowska-Curie Innovative Training Network MCnetITN3 (Grant agreement no. 722104).
Data Availability Statement This manuscript has no associated data or the data will not be deposited. [Authors' comment: Ancillary files are available together with the arXiv submission (open source).]

Open Access This article is licensed under a Creative Commons Attribution 4.0 International License, which permits use, sharing, adaptation, distribution and reproduction in any medium or format, as long as you give appropriate credit to the original author(s) and the source, provide a link to the Creative Commons licence, and indicate if changes were made. The images or other third party material in this article are included in the article's Creative Commons licence, unless indicated otherwise in a credit line to the material. If material is not included in the article's Creative Commons licence and your intended use is not permitted by statutory regulation or exceeds the permitted use, you will need to obtain permission directly from the copyright holder. To view a copy of this licence, visit http://creativecomm ons.org/licenses/by/4.0/.

Funded by $\mathrm{SCOAP}^{3}$.

\section{References}

1. ATLAS Collaboration, G. Aad et al., Observation of a new particle in the search for the Standard Model Higgs boson with the ATLAS detector at the LHC. Phys. Lett. B716, 1-29 (2012). https://doi. org/10.1016/j.physletb.2012.08.020. arXiv:1207.7214 [hep-ex]

2. C.M.S. Collaboration, S. Chatrchyan et al., Observation of a new boson at a Mass of $125 \mathrm{GeV}$ with the CMS experiment at the LHC. Phys. Lett. B 716, 30-61 (2012). https://doi.org/10.1016/j. physletb.2012.08.021. arXiv:1207.7235 [hep-ex]

3. ATLAS Collaboration, G. Aad et al., Combined measurements of Higgs boson production and decay using up to $80 \mathrm{fb}^{-1}$ of protonproton collision data at $\sqrt{s}=13 \mathrm{TeV}$ collected with the ATLAS experiment. Phys. Rev. D101(1), 012002 (2020). https://doi.org/ 10.1103/PhysRevD.101.012002. arXiv:1909.02845 [hep-ex]

4. T. Han, G. Valencia, S. Willenbrock, Structure function approach to vector boson scattering in p p collisions. Phys. Rev. Lett. 69, 3274-3277 (1992). https://doi.org/10.1103/PhysRevLett.69.3274. arXiv:hep-ph/9206246 [hep-ph]

5. T. Figy, C. Oleari, D. Zeppenfeld, Next-to-leading order jet distributions for Higgs boson production via weak boson fusion. Phys. Rev. D 68, 073005 (2003). https://doi.org/10.1103/PhysRevD.68. 073005. arXiv:hep-ph/0306109 [hep-ph]

6. E.L. Berger, J.M. Campbell, Higgs boson production in weak boson fusion at next-to-leading order. Phys. Rev. D 70, 073011 (2004). https://doi.org/10.1103/PhysRevD.70.073011. arXiv:hep-ph/0403194 [hep-ph]

7. M. Ciccolini, A. Denner, S. Dittmaier, Electroweak and QCD corrections to Higgs production via vector-boson fusion at the LHC. Phys. Rev. D 77, 013002 (2008). https://doi.org/10.1103/ PhysRevD.77.013002. arXiv:0710.4749 [hep-ph]

8. P. Nason, C. Oleari, NLO Higgs boson production via vector-boson fusion matched with shower in POWHEG. https://doi.org/10.1007/ JHEP02(2010)037. JHEP 02, 037 (2010). arXiv:0911.5299 [hep$\mathrm{ph}]$

9. S. Platzer, S. Gieseke, Dipole showers and automated NLO matching in Herwig++. Eur. Phys. J. C 72, 2187 (2012). https://doi.org/ 10.1140/epjc/s10052-012-2187-7. arXiv:1109.6256 [hep-ph]

10. S. Frixione, P. Torrielli, M. Zaro, Higgs production through vectorboson fusion at the NLO matched with parton showers. Phys. Lett. B 726, 273-282 (2013). https://doi.org/10.1016/j.physletb.2013. 08.030. arXiv:1304.7927 [hep-ph]

11. P. Bolzoni, F. Maltoni, S.-O. Moch, M. Zaro, Higgs production via vector-boson fusion at NNLO in QCD. Phys. Rev. Lett. 105, 
011801 (2010). https://doi.org/10.1103/PhysRevLett.105.011801. arXiv:1003.4451 [hep-ph]

12. P. Bolzoni, F. Maltoni, S.-O. Moch, M. Zaro, Vector boson fusion at NNLO in QCD: SM Higgs and beyond. Phys. Rev. D 85, 035002 (2012). https://doi.org/10.1103/PhysRevD.85.035002. arXiv:1109.3717 [hep-ph]

13. M. Cacciari, F. A. Dreyer, A. Karlberg, G. P. Salam, G. Zanderighi, Fully differential vector-boson-fusion higgs production at nextto-next-to-leading order. Phys. Rev. Lett. 115(8), 082002 (2015). https://doi.org/10.1103/PhysRevLett.115.082002, https://doi.org/ 10.1103/PhysRevLett.120.139901. arXiv:1506.02660 [hep-ph]. [Erratum: Phys. Rev. Lett.120,no.13,139901(2018)]

14. J. Cruz-Martinez, T. Gehrmann, E.W.N. Glover, A. Huss, Secondorder QCD effects in Higgs boson production through vector boson fusion. Phys. Lett. B 781, 672-677 (2018). https://doi.org/10.1016/ j.physletb.2018.04.046. arXiv:1802.02445 [hep-ph]

15. F. A. Dreyer, A. Karlberg, Vector-boson fusion Higgs production at three loops in QCD. Phys. Rev. Lett. 117(7), 072001 (2016) arXiv:1606.00840 [hep-ph]

16. R.V. Harlander, J. Vollinga, M.M. Weber, Gluon-induced weak boson fusion. Phys. Rev. D 77, 053010 (2008). https://doi.org/10. 1103/PhysRevD.77.053010. arXiv:0801.3355 [hep-ph]

17. T. Liu, K. Melnikov, A. A. Penin, Nonfactorizable QCD effects in Higgs boson production via vector boson fusion. arXiv:1906.10899 [hep-ph]

18. B. Jäger, F. Schissler, D. Zeppenfeld, Parton-shower effects on Higgs boson production via vector-boson fusion in association with three jets. JHEP 07, 125 (2014). https://doi.org/10.1007/ JHEP07(2014)125. arXiv:1405.6950 [hep-ph]

19. J. Alwall, R. Frederix, S. Frixione, V. Hirschi, F. Maltoni, O. Mattelaer, H.S. Shao, T. Stelzer, P. Torrielli, M. Zaro, The automated computation of tree-level and next-to-leading order differential cross sections, and their matching to parton shower simulations. JHEP 07, 079 (2014). https://doi.org/10.1007/JHEP07(2014)079. arXiv:1405.0301 [hep-ph]

20. R. Frederix, S. Frixione, V. Hirschi, D. Pagani, H.S. Shao, M. Zaro, The automation of next-to-leading order electroweak calculations. JHEP 07, 185 (2018). https://doi.org/10.1007/JHEP07(2018)185. arXiv:1804.10017 [hep-ph]

21. J. Bellm et al., Herwig 7.0/Herwig++ 3.0 release note. Eur. Phys. J. C76(4), 196 (2016). https://doi.org/10.1140/epjc/ s10052-016-4018-8. arXiv:1512.01178 [hep-ph]

22. J. Bellm et al., Herwig 7.1 Release Note. arXiv:1705.06919 [hep$\mathrm{ph}]$

23. K. Arnold et al., VBFNLO: a parton level Monte Carlo for processes with electroweak bosons. Comput. Phys. Commun. 180, 1661-1670 (2009). https://doi.org/10.1016/j.cpc.2009.03. 006. arXiv:0811.4559 [hep-ph]

24. J. Baglio et al., VBFNLO: a parton level Monte Carlo for processes with electroweak bosons-manual for version 2.7.0. arXiv:1107.4038 [hep-ph]

25. F. Campanario, T. M. Figy, S. Plätzer, M. Sjödahl, Electroweak Higgs boson plus three jet production at next-to-leading-order QCD. Phys. Rev. Lett. 111(21), 211802 (2013). https://doi.org/ 10.1103/PhysRevLett.111.211802. arXiv:1308.2932 [hep-ph]

26. S. Frixione, B.R. Webber, Matching NLO QCD computations and parton shower simulations. JHEP 06, 029 (2002). https:// doi.org/10.1088/1126-6708/2002/06/029. arXiv:hep-ph/0204244 [hep-ph]

27. S. Frixione, Z. Kunszt, A. Signer, Three jet cross-sections to next-to-leading order. Nucl. Phys. B467, 399-442 (1996). https:// doi.org/10.1016/0550-3213(96)00110-1. arXiv:hep-ph/9512328 [hep-ph]

28. S. Frixione, A general approach to jet cross-sections in QCD. Nucl. Phys. B507, 295-314 (1997). https://doi.org/10.1016/ S0550-3213(97)00574-9. arXiv:hep-ph/9706545 [hep-ph]
29. R. Frederix, S. Frixione, F. Maltoni, T. Stelzer, Automation of nextto-leading order computations in QCD: the FKS subtraction. JHEP 10, 003 (2009). https://doi.org/10.1088/1126-6708/2009/10/003. arXiv:0908.4272 [hep-ph]

30. R. Frederix, S. Frixione, A.S. Papanastasiou, S. Prestel, P. Torrielli, Off-shell single-top production at NLO matched to parton showers. JHEP 06, 027 (2016). https://doi.org/10.1007/JHEP06(2016)027. arXiv:1603.01178 [hep-ph]

31. G. Ossola, C. G. Papadopoulos, R. Pittau, Reducing full one-loop amplitudes to scalar integrals at the integrand level. Nucl. Phys. B763, 147-169 (2007). https://doi.org/10.1016/j.nuclphysb.2006. 11.012. arXiv:hep-ph/0609007 [hep-ph]

32. P. Mastrolia, E. Mirabella, T. Peraro, Integrand reduction of one-loop scattering amplitudes through Laurent series expansion. JHEP 06, 095 (2012). https://doi.org/10.1007/JHEP06(2012)095. arXiv:1203.0291 [hep-ph]. [Erratum: JHEP11,128(2012)]

33. G. Passarino, M.J.G. Veltman, One loop corrections for e+ e- Annihilation Into mu+ mu- in the Weinberg Model. Nucl. Phys. B 160, 151-207 (1979). https://doi.org/10.1016/0550-3213(79)90234-7

34. A.I. Davydychev, A simple formula for reducing Feynman diagrams to scalar integrals. Phys. Lett. B 263, 107-111 (1991). https://doi.org/10.1016/0370-2693(91)91715-8

35. A. Denner, S. Dittmaier, Reduction schemes for one-loop tensor integrals. Nucl. Phys. B734, 62-115 (2006). https://doi.org/10. 1016/j.nuclphysb.2005.11.007. arXiv:hep-ph/0509141 [hep-ph]

36. V. Hirschi, R. Frederix, S. Frixione, M.V. Garzelli, F. Maltoni, R. Pittau, Automation of one-loop QCD corrections. JHEP 05, 044 (2011). https://doi.org/10.1007/JHEP05(2011)044. arXiv:1103.0621 [hep-ph]

37. G. Ossola, C.G. Papadopoulos, R. Pittau, CutTools: a program implementing the OPP reduction method to compute oneloop amplitudes. JHEP 03, 042 (2008). https://doi.org/10.1088/ 1126-6708/2008/03/042. arXiv:0711.3596 [hep-ph]

38. T. Peraro, Ninja: automated integrand reduction via laurent expansion for one-loop amplitudes. Comput. Phys. Commun. 185, 2771-2797 (2014). https://doi.org/10.1016/j.cpc.2014.06. 017. arXiv:1403.1229 [hep-ph]

39. V. Hirschi, T. Peraro, Tensor integrand reduction via Laurent expansion. JHEP 06, 060 (2016). https://doi.org/10.1007/ JHEP06(2016)060. arXiv:1604.01363 [hep-ph]

40. H.-S. Shao, Iregi user manual (unpublished)

41. A. Denner, S. Dittmaier, L. Hofer, Collier: a fortran-based complex one-loop library in extended regularizations. Comput. Phys. Commun. 212, 220-238 (2017). https://doi.org/10.1016/j.cpc.2016.10. 013. arXiv:1604.06792 [hep-ph]

42. F. Cascioli, P. Maierhofer, S. Pozzorini, Scattering amplitudes with open loops. Phys. Rev. Lett. 108, 111601 (2012). https://doi.org/ 10.1103/PhysRevLett.108.111601. arXiv:1111.5206 [hep-ph]

43. R. Frederix, S. Frixione, V. Hirschi, F. Maltoni, R. Pittau, P. Torrielli, Four-lepton production at hadron colliders: aMC@NLO predictions with theoretical uncertainties. JHEP 02, 099 (2012). https://doi.org/10.1007/JHEP02(2012)099. arXiv: 1110.4738 [hep-ph]

44. A. Ballestrero et al., Precise predictions for same-sign W-boson scattering at the LHC. Eur. Phys. J. C78(8), 671 (2018). https://doi. org/10.1140/epjc/s10052-018-6136-y. arXiv:1803.07943 [hep$\mathrm{ph}]$

45. T. Sjöstrand, S. Ask, J. R. Christiansen, R. Corke, N. Desai, P. Ilten, S. Mrenna, S. Prestel, C. O. Rasmussen, P. Z. Skands, An introduction to PYTHIA 8.2. Comput. Phys. Commun. 191, 159-177 (2015). https://doi.org/10.1016/j.cpc.2015.01.024. arXiv: 1410.3012 [hep-ph]

46. J. Bellm et al., Herwig 7.2 Release Note. arXiv:1912.06509 [hep$\mathrm{ph}$ ]

47. S. Alioli, P. Nason, C. Oleari, E. Re, A general framework for implementing NLO calculations in shower Monte Carlo programs: 
the POWHEG BOX. JHEP 06, 043 (2010). https://doi.org/10.1007/ JHEP06(2010)043. arXiv:1002.2581 [hep-ph]

48. P. Nason, A new method for combining NLO QCD with shower Monte Carlo algorithms. JHEP 11, 040 (2004). https://doi.org/10. 1088/1126-6708/2004/11/040. arXiv:hep-ph/0409146 [hep-ph]

49. S. Frixione, P. Nason, C. Oleari, Matching NLO QCD computations with Parton Shower simulations: the POWHEG method. JHEP 11, 070 (2007). https://doi.org/10.1088/1126-6708/2007/11/070. arXiv:0709.2092 [hep-ph]

50. T. Figy, V. Hankele, D. Zeppenfeld, Next-to-leading order QCD corrections to Higgs plus three jet production in vector-boson fusion. JHEP 02, 076 (2008). https://doi.org/10.1088/1126-6708/ 2008/02/076. arXiv:0710.5621 [hep-ph]

51. M. Bahr et al., Herwig++ physics and manual. Eur. Phys. J. C 58, 639-707 (2008). https://doi.org/10.1140/epjc/ s10052-008-0798-9. arXiv:0803.0883 [hep-ph]

52. S. Gieseke, P. Stephens, B. Webber, New formalism for QCD parton showers. JHEP 12, 045 (2003). https://doi.org/10.1088/ 1126-6708/2003/12/045. arXiv:hep-ph/0310083 [hep-ph]

53. S. Platzer, S. Gieseke, Coherent parton showers with local recoils. JHEP 01, 024 (2011). https://doi.org/10.1007/JHEP01(2011)024. arXiv:0909.5593 [hep-ph]

54. J. Baglio et al., Release Note-VBFNLO 2.7.0. arXiv:1404.3940 [hep-ph]

55. J. Bellm, G. Nail, S. Plätzer, P. Schichtel, A. Siódmok, Parton shower uncertainties with Herwig 7: benchmarks at leading order. Eur. Phys. J. C76(12), 665 (2016). https://doi.org/10.1140/epjc/ s10052-016-4506-x. arXiv:1605.01338 [hep-ph]

56. K. Cormier, S. Plätzer, C. Reuschle, P. Richardson, S. Webster, Parton showers and matching uncertainties in top quark pair production with Herwig 7. Eur. Phys. J. C79(11), 915, (2019). https://doi.org/ 10.1140/epjc/s10052-019-7370-7. arXiv:1810.06493 [hep-ph]

57. B. Cabouat, T. Sjstrand, Some dipole shower studies. Eur. Phys. J. C78(3), 226 (2018). https://doi.org/10.1140/epjc/ s10052-018-5645-z. arXiv:1710.00391 [hep-ph]

58. R. Frederix, S. Frixione, S. Prestel, P. Torrielli, On the reduction of negative weights in MC@NLO-type matching procedures. arXiv:2002.12716 [hep-ph]

59. CMS Collaboration, A. M. Sirunyan et al., Electroweak production of two jets in association with a $\mathrm{Z}$ boson in protonproton collisions at $\sqrt{s}=13 \mathrm{TeV}$. Eur. Phys. J. C78(7), 589 (2018). https://doi.org/ 10.1140/epjc/s10052-018-6049-9. arXiv:1712.09814 [hep-ex]
60. J. Butterworth et al., PDF4LHC recommendations for LHC Run II. J. Phys. G43, 023001 (2016). https://doi.org/10.1088/0954-3899/ 43/2/023001. arXiv:1510.03865 [hep-ph]

61. A. Buckley, J. Ferrando, S. Lloyd, K. Nordström, B. Page, M. Rüfenacht, M. Schönherr, G. Watt, LHAPDF6: parton density access in the LHC precision era. Eur. Phys. J. C 75, 132 (2015). https://doi. org/10.1140/epjc/s10052-015-3318-8. arXiv:1412.7420 [hep-ph]

62. M. Cacciari, G.P. Salam, G. Soyez, The anti- $k_{t}$ jet clustering algorithm. JHEP 04, 063 (2008). https://doi.org/10.1088/1126-6708/ 2008/04/063. arXiv:0802.1189 [hep-ph]

63. M. Cacciari, G.P. Salam, G. Soyez, FastJet user manual. Eur. Phys. J. C72, 1896 (2012). https://doi.org/10.1140/epjc/ s10052-012-1896-2. arXiv:1111.6097 [hep-ph]

64. E. Bagnaschi, F. Maltoni, A. Vicini, M. Zaro, Lepton-pair production in association with a $b \bar{b}$ pair and the determination of the $W$ boson mass. JHEP 07, 101 (2018). https://doi.org/10.1007/ JHEP07(2018)101. arXiv:1803.04336 [hep-ph]

65. M. Rauch, S. Plätzer, Parton shower matching systematics in vector-boson-fusion WW production. Eur. Phys. J. C 77(5), 293 (2017). https://doi.org/10.1140/epjc/s10052-017-4860-3. arXiv:1802.09955 [hep-ph]

66. F. Campanario, T. M. Figy, S. Plätzer, M. Rauch, P. Schichtel, M. Sjödahl, Stress testing the vector-boson-fusion approximation in multijet final states. Phys. Rev. D98(3), 033003 (2018), https://doi.org/10.1103/PhysRevD.98.033003. arXiv:1802.09955 [hep-ph]

67. M. Rauch, D. Zeppenfeld, Jet clustering dependence of Higgs boson production in vector-boson fusion. Phys. Rev. D95(11), 114015 (2017). https://doi.org/10.1103/PhysRevD.95. 114015. arXiv:1703.05676 [hep-ph]

68. J. Bellm et al., Jet cross sections at the LHC and the quest for higher precision. Eur. Phys. J. C80(2), 93 (2020). https://doi.org/10.1140/ epjc/s10052-019-7574-x. arXiv:1903.12563 [hep-ph]

69. S. Amoroso et al., Les Houches 2019: Physics at TeV Colliders: Standard Model Working Group Report. in 11th Les Houches Workshop on Physics at TeV Colliders: PhysTeV Les Houches (PhysTeV 2019) Les Houches, France, June 10-28, 2019. (2020). arXiv:2003.01700 [hep-ph]

70. C. Bierlich et al., Robust independent validation of experiment and theory: Rivet version 3. SciPost Phys. 8, 026 (2020). https://doi. org/10.21468/SciPostPhys.8.2.026. arXiv:1912.05451 [hep-ph] 